Pitch and roll sensing using fibre Bragg gratings in multicore fibre

This article has been downloaded from IOPscience. Please scroll down to see the full text article.

2004 Meas. Sci. Technol. 151642

(http://iopscience.iop.org/0957-0233/15/8/036)

View the table of contents for this issue, or go to the journal homepage for more

Download details:

IP Address: 130.159.179.120

The article was downloaded on 06/02/2013 at 15:47

Please note that terms and conditions apply. 


\title{
Pitch and roll sensing using fibre Bragg gratings in multicore fibre
}

\author{
William N MacPherson ${ }^{1}$, Gordon M H Flockhart ${ }^{1,3}$, \\ Robert R J Maier ${ }^{1}$, James S Barton ${ }^{1}$, Julian D C Jones ${ }^{1}$, \\ Donghui Zhao ${ }^{2}$, Lin Zhang ${ }^{2}$ and Ian Bennion ${ }^{2}$ \\ ${ }^{1}$ School of Engineering and Physical Sciences, Heriot-Watt University, \\ Edinburgh EH14 4AS, UK \\ ${ }^{2}$ Photonics Research Group, Electronic Engineering, Aston University, Aston Triangle, \\ Birmingham B4 7ET, UK \\ E-mail: W.N.MacPherson@hw.ac.uk
}

Received 22 December 2003

Published 19 July 2004

Online at stacks.iop.org/MST/15/1642

doi:10.1088/0957-0233/15/8/036

\begin{abstract}
We report the first use of a multicore fibre incorporating fibre Bragg grating strain sensors in each core as a fibre optic pitch and roll sensor. A length of four-core fibre supported at one end forms a cantilever. The differential strains between opposite grating pairs depend on the fibre's orientation in pitch (in the vertical plane) and roll (azimuth) with respect to gravity. Resolutions of $\pm 2^{\circ}$ in roll and $\pm 15^{\circ}$ in pitch were measured.
\end{abstract}

Keywords: bend measurement, orientation measurement, fibre Bragg grating, fibre optic sensing, multicore fibre

\section{Introduction}

The objective of this paper is to describe an optical 'attitude sensor', measuring pitch and roll angles of the sensor by the elastic deformation produced by gravitational force acting on an optical fibre cantilever. The fibre has multiple single-mode cores and the deflection is measured via the strain induced in Bragg gratings written into the cores. Apart from the well-known advantages of using an optical fibre sensor, our design also has the desirable attribute of being based on the elastic properties of the fused silica from which the fibre is made, which are near-ideal. A compact optical sensor for orientation measurement has applications to industrial processes in hazardous environments and to shape monitoring in flexible structures such as towed hydrophone arrays.

Fibre Bragg gratings (FBG) have been extensively demonstrated for use as sensors [1]. A FBG is inherently sensitive to temperature and strain via the thermo-optic effect strain-optic effects and changes in the physical dimensions of the fibre. This sensitivity to both strain and temperature often poses a problem in practical sensing applications. One

3 Present address: Naval Research Laboratory, 4555 Overlook Avenue SW, Washington, DC 20375-5338, USA technical solution is to make two measurements, using two FBGs, one of which is mounted in such a way as to isolate it from one of the measurands. For example, a grating may be mounted loosely inside a protective capillary to isolate it from strain and thus measure temperature only. Similarly, a grating may be mounted such that it acts as two gratings by pre-straining and securing one half to the structure, leaving the unsecured section effectively strain-isolated [2]. Alternatively, two gratings may be mounted such that one of the measurands (typically temperature) is the common mode, in which case a differential wavelength measurement will yield the strain. For example two gratings on either side of a cantilever beam can be isothermal, but will measure differential strain as a function of the cantilever deflection [3].

In order for the common mode compensation technique to work, it is crucial that each grating is at the same temperature. In order to achieve this thermal equilibrium it is beneficial to locate the gratings as close together as possible in a homogeneous structure. Multicore fibre containing gratings at the same axial position fulfils this requirement in a compact package ideally suited to curvature measurement. We have previously presented results in which a multicore fibre (MCF) is demonstrated for bending in one dimension [4], and in two dimensions [5]. Other configurations using separate 


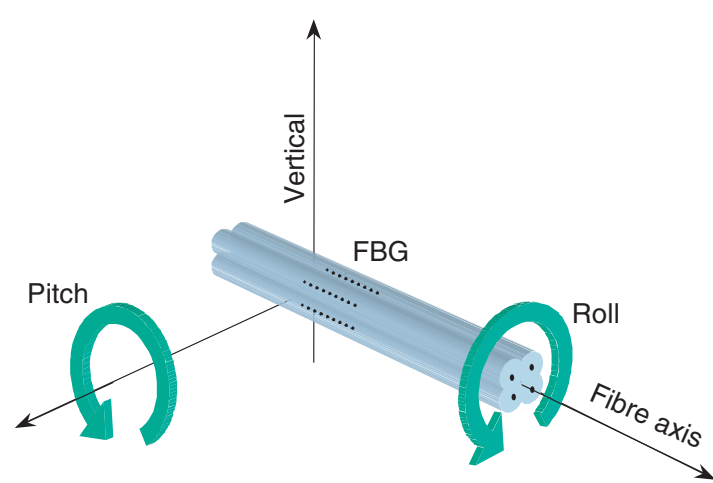

Figure 1. Definition of axes and pitch $(\theta)$ and roll $(\phi)$ angles.

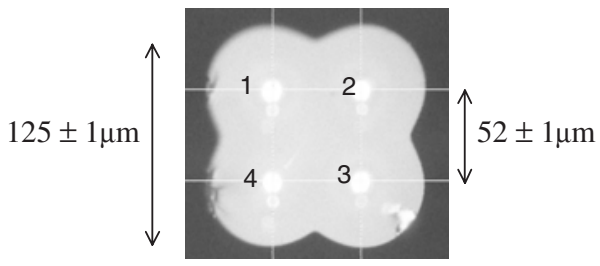

Figure 2. Multicore fibre used in the attitude sensor.

fibres [6] and photonic crystal fibre (PCF) [7] have also been reported. These techniques for curvature measurement, especially those using the multicore fibre, are particularly useful for practical applications because they are inherently temperature insensitive. In multicore fibre the sensing cores are close together in one continuous material, promoting good thermal contact.

Furthermore, for our application of a pitch and roll sensor (or attitude sensor), it is desirable to produce a miniaturized sensor with minimum hysteresis, as is the case for the elastic properties of the fused silica fibre.

\section{Pitch and roll measurement}

We report here the functional principle of an attitude or orientation sensor capable of measuring pitch and roll by means of a four-core fibre curvature sensor. Figure 1 defines the geometry of the roll and pitch angles.

The four-core fibre was supported at one end and the resulting cantilever acted upon by gravity, which bends the free end of the fibre downwards. A small mass attached to the end of the fibre further increased the bend, thus improving the sensitivity. The FBGs in the MCF were located close to the support, to experience the maximum differential strain. The neutral axis of the cantilever lies within the horizontal plane tangential to the fibre at any point along its length. For any arbitrary roll angle, the gratings will be subjected to differential axial strains proportional to their distances above or below the plane of the neutral axis. In this configuration we obtain the angle of roll by comparing the ratio of the strain differences in two orthogonal axes with respect to the fibre, i.e. core pairs $(1,2)$ and $(2,3)$, as shown in figure 2. A measure of the pitch angle can be obtained from the magnitude of the differential strain between pairs. If we denote the wavelength separation associated with grating pair $(1,2)$ as $x$, and that associated with the orthogonal pair $(2,3)$ as $y$, then the roll $(\phi)$ and pitch $(\theta)$ angles are given by

$$
\begin{gathered}
\phi=\tan ^{-1}\left[\frac{y}{x}\right] \\
\vartheta=\cos ^{-1}\left[\frac{\sqrt{x^{2}+y^{2}}}{\sqrt{x_{0}^{2}+y_{0}^{2}}}\right]
\end{gathered}
$$

where $x_{0}, y_{0}$ denote the values of $x, y$ at $\theta=0$. These simple expressions will apply in the special case where all the grating wavelengths are identical when curvature is zero. In practice this is not the case and the appropriate offsets must be subtracted from the values of $x$ and $y$.

\section{Experimental configuration}

A photograph of the cross-section of the multicore fibre used is given in figure 2. The fibre consists of four cores arranged in a square array with a core separation of $52 \pm 1 \mu \mathrm{m}$, within an envelope $125 \mu \mathrm{m}$ square with a maximum outer diameter of $146 \pm 1 \mu \mathrm{m}$. The MCF was photosensitized by hydrogen loading and fibre Bragg gratings were written into four cores by exposure through a phase mask with a $255 \mathrm{~nm}$ frequencydoubled copper-vapour laser. The reflectivities of the gratings differ due to the rotational alignment of the fibre relative to the UV laser beam during writing.

For the pitch and roll sensing application, optimum results would be obtained by using narrow bandwidth, Gaussian profile grating spectra. The grating spectra in figure 3 depart from this ideal as the square geometry of the cores and the profile of the cladding act to distort the writing beam resulting in the complex grating structures implied by the observed reflection spectra. However, with better rotational control of the fibre during the writing process, and possible masking to ensure only one core is exposed at a time, it is anticipated that the grating spectra could be improved. Although four gratings were written, the three best were selected for use in these experiments, as the minimum requirement to form two orthogonal pairs. If four consistent narrow grating spectra are available, the sensor offers redundancy in the choice of grating pairs.

A multicore to single-core fibre fan-out was fabricated to interrogate each core of the MCF independently. It was constructed by reducing the diameter of four single-core singlemode fibres to the core spacing of the MCF using hydrofluoric acid and arranging them in a square shape within a temporary splice ferrule to match the MCF core spacing. The $2 \times 2$ array of etched and polished fibres was butt-coupled to the end face of the MCF and secured by UV curing epoxy after optimizing the angular alignment. The insertion losses of the resulting joint for each core were measured to be 0.2 , $1.2,1.6$ and $3.4 \mathrm{~dB}$ (limited by the accuracy of the etching). Cross-talk between cores, maximum $-60 \mathrm{~dB}$, was negligible for the purposes of these measurements. The MCF containing the sensor gratings was connected to the fan-out MCF by an adhesive bonded splice.

The complete interrogation scheme is shown in figure 4 . The tunable laser input light was fanned out by a combination of couplers, circulators and isolators into five channels comprising conventional single-mode fibres. Four of these 

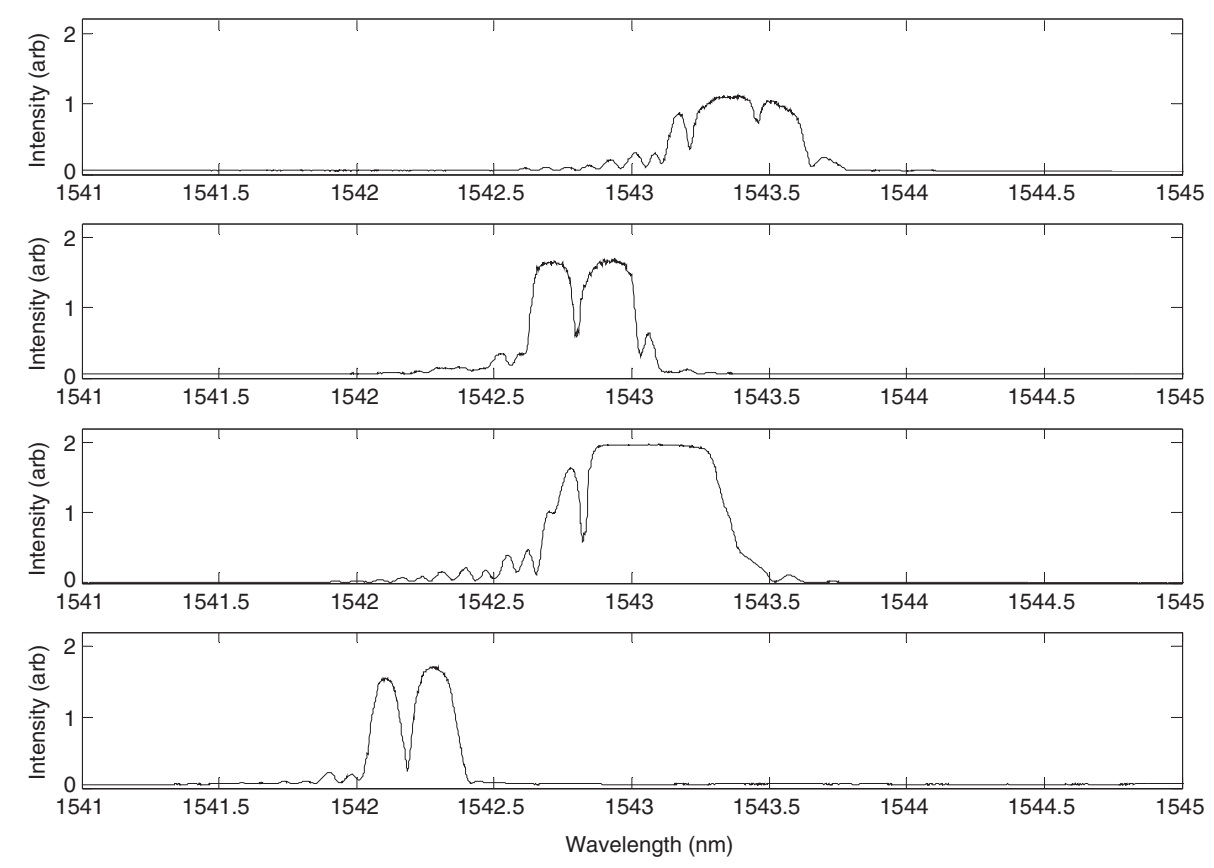

Figure 3. Reflection spectra of the four FBGs used for measurement of cantilever curvature.

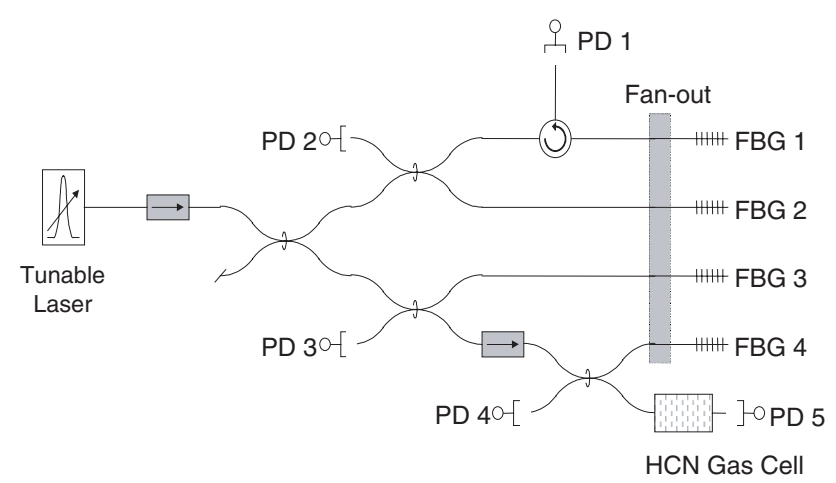

Figure 4. Interrogation scheme incorporating four-core fan out and coupler/circulator tree network. A gas reference cell is included to enable absolute wavelength measurement and to enable compensation for variations in the tunable laser scan speed.

were the input fibres to the single-core to multicore fibre fan-out. The reflected light from each core was incident on four photodetectors (PD1-4) with negligible cross-talk between these channels due to the high isolation efficiency of the isolator and circulator $(>45 \mathrm{~dB})$. The remaining channel passed through a calibration gas cell containing hydrogen cyanide, which has a well-characterized series of absorption lines in the range 1540-1544 nm, covering the wavelengths of the sensor gratings. The gas cell transmission spectrum was recorded alongside the grating spectra, and was used to compensate for wavelength drift and variations in the scan speed of the tunable laser source, scanning at $\sim 1 \mathrm{~nm} \mathrm{~s}^{-1}$. It is important to note that the fibre network was specifically designed to isolate the laser source from the reflected optical signal, thus obtaining maximum laser stability. In practice no effects were observed that could be traced to feedback instability.

The optical interrogation system allowed all four gratings and the reference channel to be recorded simultaneously.

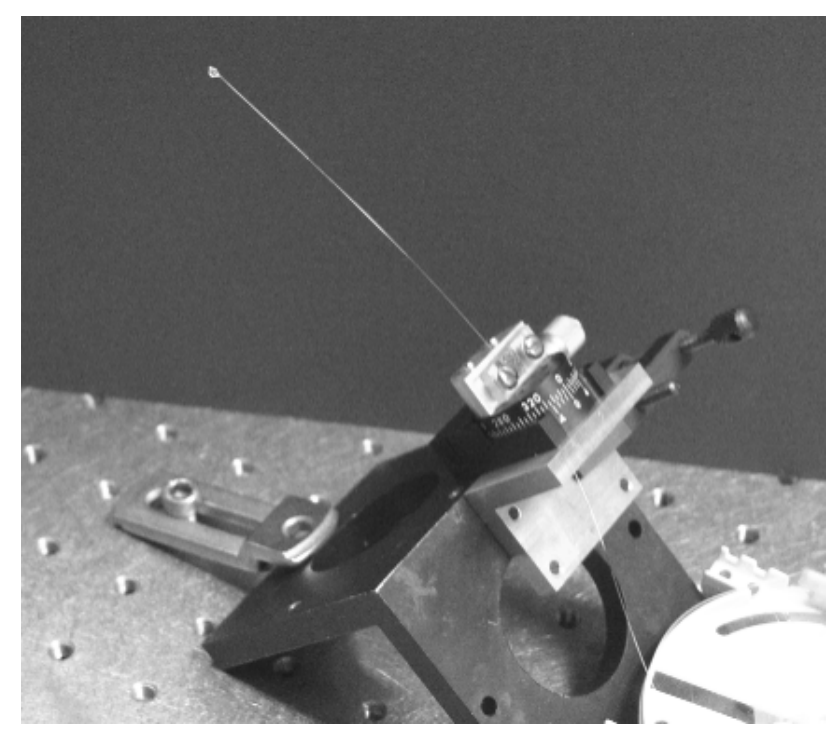

Figure 5. Sensing fibre mounted in rotary stage; in this case the pitch angle is set at $45^{\circ}$.

In our experiment the bandwidth was limited to $\sim 0.25 \mathrm{~Hz}$ due to analogue-to-digital conversion limitations; however, it is anticipated that this could be increased by an order of magnitude by using alternative $\mathrm{A} / \mathrm{D}$ systems.

The sensing element is shown in figure 5 . The multicore fibre cantilever was approximately $90 \mathrm{~mm}$ long, fixed to a rotary stage which is capable of rotating the fibre through $>360^{\circ}$ with better than $1^{\circ}$ accuracy and repeatability, to impart the 'roll'. This stage was mounted at several angles of pitch $0^{\circ}, 30^{\circ}, 45^{\circ}, 60^{\circ}$ and $75^{\circ}$ above the horizontal, and the wavelengths from each grating were recorded for a range of roll from $-90^{\circ}$ to $+450^{\circ}$ (i.e. $1 \frac{1}{2}$ turns). A small mass of epoxy $(5 \pm 1 \mathrm{mg})$ was attached to the free end of the cantilever to increase the bending under gravity. The coating was retained 


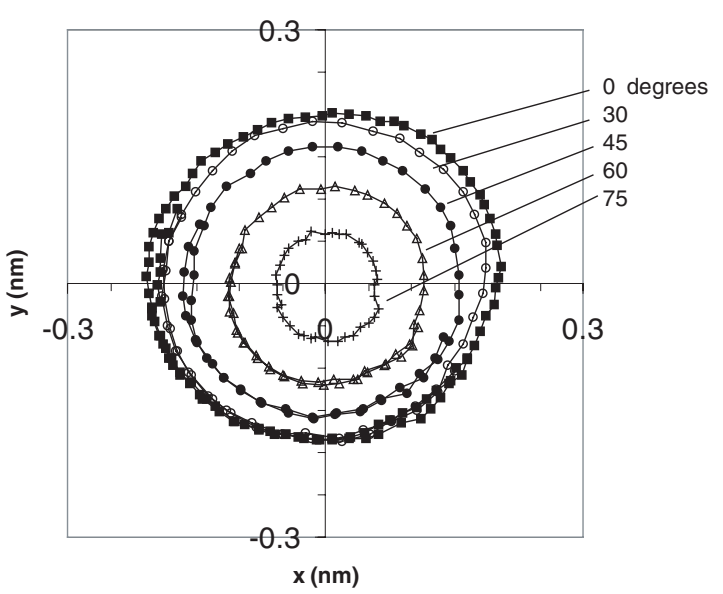

Figure 6. Experimental results from pitch $(\theta)$ and roll $(\phi)$ measurements. $x=\lambda_{1}-\lambda_{2}, y=\lambda_{2}-\lambda_{3}$ are the differential Bragg wavelength shifts (in nm with offset removed) between orthogonal pairs $(1,2)$ and $(2,3)$ of MCF gratings. Data are plotted for $\theta=0^{\circ}$, $30^{\circ}, 45^{\circ}\left(\phi\right.$ range 0 to $540^{\circ}$ ) and $\theta=60^{\circ}, 75^{\circ}\left(\phi\right.$ range 0 to $360^{\circ}$ ).

on the final $30 \mathrm{~mm}$ of fibre, which increased the effective mass to $6.2 \pm 1 \mathrm{mg}$.

The scanning tunable laser enabled the grating spectra to be measured at each angular setting. For simplicity we performed a peak fitting routine to the grating spectrum to find an effective 'centre wavelength' using a second-order polynomial fit to a region above a specified threshold. This simple means of determining the grating wavelength was found to be repeatable to better than $6 \mathrm{pm}$, which was more than adequate for these trial experiments.

The grating centre wavelengths taken as pairs $(1,2)$ and $(2,3)$ were used to determine $x$ and $y$ in equations (1) and (2). An $x-y$ plot can be used to represent the results graphically as the roll was varied from 0 to $360^{\circ}$. If the data points follow equation (1) their locus will be a circle, the radius of which will be a function of the pitch according to equation (2). The results are plotted in figure 6 , in which the pitch was set at $0^{\circ}$, $30^{\circ}, 45^{\circ}, 60^{\circ}$ and $75^{\circ}$ above the horizontal.

The repeatability of these results was tested over a subrange of the measurements: the outer three sets of results $\left(0^{\circ}\right.$, $30^{\circ}$ and $45^{\circ}$ pitch results) have a $180^{\circ}$ section repeated, which shows good overlap.

\section{Discussion}

The circular regions defined by the data points in figure 6 are fairly uniform except for the $\theta=75^{\circ}$ data, where there is some distortion evident as the roll angle is varied. We attribute this to the effect of the non-circular cross-section of the MCF becoming more important relative to the reduced component of weight perpendicular to the fibre at a high pitch angle. The roll accuracy was determined to be $\pm 2^{\circ}$, which could be improved by using narrower gratings to improve wavelength measurement and adding further mass to the cantilever to increase curvature.

The accuracy of the pitch measurement is less well defined, and is a function of the pitch. At large pitch angles the sensor loses sensitivity, due to small cantilever deviation at $\geqslant 80^{\circ}$ below horizontal and due to mechanical instability

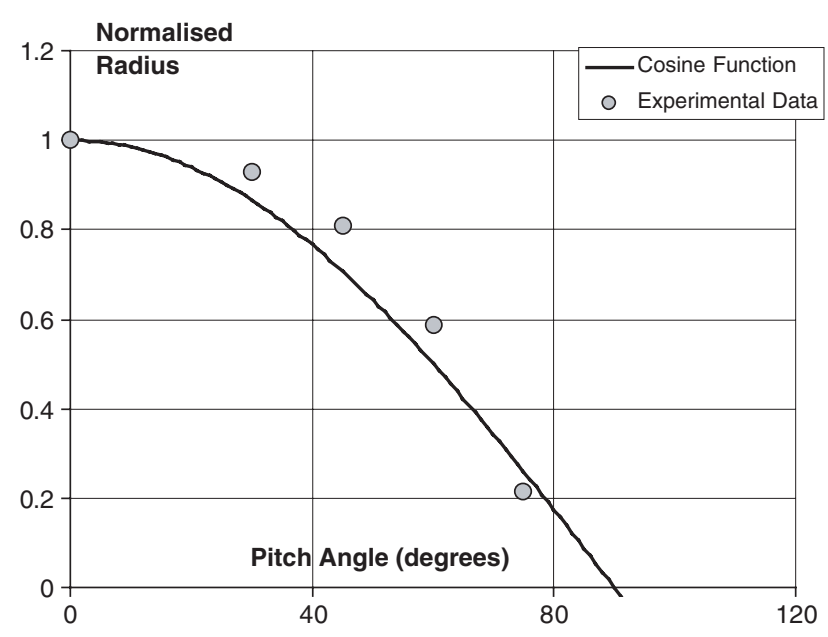

Figure 7. Normalized strain amplitude (i.e. radius of the data circles in figure 6) versus pitch angle. A cosine curve (equation (2)) is shown for comparison.

$\geqslant 80^{\circ}$ above horizontal. However, we estimate that the current measurement gives pitch to better than $\pm 15^{\circ}$ over the range $\theta=0$ to $75^{\circ}$. The normalized radii of the circles in figure 6 are plotted against pitch angle in figure 7 . Relative to the $\cos \theta$ curve, the measured strains remain slightly higher and then fall more rapidly with pitch angle.

The differential strain corresponding to the data in figure 6 can be estimated from the axial strain sensitivity of $0.997 \mathrm{pm} \mu \varepsilon^{-1}$ that we have previously measured [4] in similar MCF. The diameter of the $\theta=0$ circle is $394 \mathrm{pm}$ in wavelength shift between the grating pairs, or an observed differential strain of $395 \mu \varepsilon$. The expected strain can be estimated to a first approximation by applying the standard equations for a uniform cantilever to a circular cross-section fibre of the equivalent area. From the MCF shape in figure 2, an equivalent area circular fibre is $125 \times 1.060=132.5 \mu \mathrm{m}$ diameter. There are two contributions to the deflection of the fibre cantilever: loading due to the mass per unit length and loading from the mass at the fibre end. Consider the fibre to be clamped at $z=0$, extending to $z=L$, with the gratings located at $+z_{g}$. From simple beam theory a linear normal stress gradient is set up across the fibre, due to the bending of the fibre. Denoting the radius of curvature at $z_{g}$ as $R$, the difference in axial strains between a grating pair $(1,2)$ separated by distance $d$ is [4]

$$
\Delta \varepsilon=\varepsilon_{1}-\varepsilon_{2}=\frac{d}{R} .
$$

The radius of curvature $R$ can be evaluated for the loading from the mass per unit length and from the end loading, and the two contributions to $\Delta \varepsilon$ added as follows [8]:

$$
\begin{gathered}
R_{\mathrm{ml}}=\frac{E I}{w}\left[L z_{g}-\frac{1}{2} z_{g}^{2}-\frac{1}{2} L^{2}\right]^{-1} \\
R_{\mathrm{el}}=\frac{E I}{F}\left[z_{g}-L\right] \\
\Delta \varepsilon=d\left[\frac{1}{R_{\mathrm{ml}}}+\frac{1}{R_{\mathrm{el}}}\right]
\end{gathered}
$$

where $E$ is the Young's modulus of the fibre, $I$ is the second moment of the cross-sectional area $\left(=\pi D^{4} / 64\right.$ for a circular 
cylinder diameter $D), w$ is the fibre weight per unit length and $F$ is the applied load at the fibre end. We model the fibre used in the experiments as $E=72 \mathrm{GPa}, D=132.5 \mu \mathrm{m}, z_{g}=$ $5 \mathrm{~mm}, L=90 \mathrm{~mm}, w=2.97 \times 10^{-4} \mathrm{~N} \mathrm{~m}^{-1}, F=6.7 \times 10^{-5} \mathrm{~N}$ and $d=52 \mu \mathrm{m}$. The resulting differential strain between a grating pair is calculated to be $329 \mu \varepsilon$, to be compared with the observed maximum value of $395 \mu \varepsilon$ in the horizontal $(\theta=0)$ orientation. The agreement is considered reasonable given the simplifying approximations and uncertainties in the parameters.

We can use this model to propose a more ruggedly packaged sensor for practical application, in which a shorter length of fibre is clamped and located coaxially within an oil-filled cylinder. Viscous damping will reduce vibration sensitivity and reduce the risk of fibre breakage. A fibre $45 \mathrm{~mm}$ in length loaded with a mass of $20 \mathrm{mg}$ (making suitable allowance for buoyancy in the oil) would generate a strain difference of $400 \mu \varepsilon$ for performance comparable to that reported above. A cylinder $50 \mathrm{~mm}$ in length and $20 \mathrm{~mm}$ internal diameter would accommodate the fibre up to its maximum deflection of $6 \mathrm{~mm}$ at zero pitch angle.

Suitable application areas for a compact, fibre-linked orientation sensor are flexible compliant structures, for example robot arms and antennae such as underwater hydrophone arrays [9] or steerable telescopes. Fibre technology is well suited to large structures requiring long downleads and to specialist robots for use in hazardous environments ranging from nuclear decommissioning to mine clearance and underwater salvage. A particular advantage of the multicore design is its ability to multiplex the orientation sensor with one or more additional sets of FBGs as twodimensional multicore grating bend sensors [5] along a single fibre which can be embedded in a flexible structural element.

One significant improvement would be the ability to write better-defined gratings in the MCF. The gratings used in this experiment contain a great deal of structure and are rather wide (up to $\sim 1.0 \mathrm{~nm}$ ): narrower gratings would enable more reliable and accurate determination of the grating wavelength. Currently we are investigating improvements to the writing procedure, including on-line monitoring of the MCF during the writing process, and improvements in the MCF positional and rotational alignment. Preliminary results indicate that it is possible to write improved gratings in MCF with better-defined spectral peaks $\sim 250 \mathrm{pm}$ wide, allowing a reduction of a factor of $\sim 5$ in the uncertainty in peak wavelength determination, i.e. resolutions of $\pm 0.4^{\circ}$ in roll and $\pm 3^{\circ}$ in pitch. We are also investigating alternative multicore fibres with circular cladding profiles for better mechanical performance.

\section{Conclusion}

We have described a proof-of-principle experiment demonstrating a multicore fibre curvature sensor applied to pitch and roll angle measurement. The fibre, which incorporates fibre Bragg grating sensor elements, was connected to a scanning laser interrogation scheme via a purpose-built fan-out unit. In this way simultaneous measurements of all four cores of the MCF could be made with $\sim 0.25 \mathrm{~Hz}$ bandwidth. The sensor was capable of measuring roll to $\pm 2^{\circ}$, and pitch to $\pm 15^{\circ}$.

\section{Acknowledgments}

The authors would like to acknowledge the UK Engineering and Physical Science Research Council and UK Defence Science and Technology Laboratory (DSTL) for provision of funding through the Joint Grant Scheme. The authors thank France Telecom for their generosity in supplying the MCF. We also thank Ann Menim, of the Geology and Geophysics Department of the University of Edinburgh, for the use of their facilities and her guidance for hydrofluoric acid etching, and Bruce Davies of Heriot-Watt University, for useful discussions. WNM wishes to acknowledge the UK Engineering and Physical Sciences Research Council (EPSRC) for provision of funding under the Advanced Fellowship Scheme.

\section{References}

[1] Rao Y J 1997 In-fibre Bragg grating sensors Meas. Sci. Technol. 8 355-75

[2] Song M, Lee S B, Choi S S and Lee B 1998 Dynamic-strain measurement with dual-grating fiber sensor Appl. Opt. 37 3484-6

[3] Xu M G, Archambault J L, Reekie L and Dakin J P 1994 Thermally-compensated bending gauge using surface-mounted fibre gratings Int. J. Optoelec. 9 281-3

[4] Gander M J, MacPherson W N, McBride R, Jones J D C Zhang L, Bennion I, Blanchard P M, Burnett J G and Greenaway A H 2000 Bend measurement using Bragg gratings in multicore fibre Electron. Lett. 36 120-1

[5] Flockhart G M H, MacPherson W N, Barton J S, Jones J D C, Zhang L and Bennion I 2003 Two-axis bend measurement with Bragg gratings in multicore optical fiber Opt. Lett. 28 387-9

[6] Araujo F M, Ferreira L A and Santos J L 2002 Simultaneous determination of curvature, plane of curvature, and temperature by use of a miniaturized sensing head based on fiber Bragg gratings Appl. Opt. 41 2401-7

[7] MacPherson W N, Gander M J, McBride R, Jones J D C, Blanchard P M, Burnett J G, Greenaway A H, Mangan B, Birks T A, Knight J C and Russell P St J 2001 Remotely addressed optical fibre curvature sensor using multicore photonic crystal fibre Opt. Commun. 193 97-104

[8] Fenner R T 1989 Mechanics of Solids chapter 6 (Oxford: Blackwell)

[9] Cranch G A, Nash P J and Kirkendall C K 2003 Large-scale remotely interrogated arrays of fiber-optic interferometric sensors for underwater acoustic applications IEEE Sensors J. 3 19-30 